\title{
La reforma de la financiación autonómica en el marco de los nuevos Estatutos ${ }^{1}$
}

\author{
Francisco Escribano \\ Catedrático de Derecho Financiero \\ Universidad de Sevilla
}

1. Advertencia sobre el objeto: acerca del método y del interés metodológico de estas Jornadas.

2. El marco constitucional: los principios informadores y los límites del sistema de financiación autonómica. La posición del Tribunal Constitucional.

3. Los diversos modelos de financiación: de la Hacienda de transferencias al sistema de corresponsabilidad.

4. La reforma de la financiación autonómica con ocasión de los nuevos Estatutos de Autonomía: premisas.

5. El sistema y el modelo de financiación en los nuevos Estatutos.

\section{ADVERTENCIA SOBRE EL OBJETO: ACERCA DEL MÉTODO Y DEL INTERÉS METODOLÓGICO DE ESTAS JORNADAS.}

Como es sabido, la iniciativa de organizar anualmente estas Jornadas de estudio entre profesores de Derecho Financiero, fue una iniciativa de mi ami-

\footnotetext{
${ }^{1}$ Este trabajo tiene su origen en las Notas que me sirvieron para la Presentación de las IV Jornadas Jaime García-Añoveros, que tuvieron lugar en junio de 2006 en la sede del IEF del Ministerio de Hacienda, bajo el lema que da título al trabajo y que no he querido modificar, a pesar de que en su desarrollo defienda que la reforma de la financiación no necesariamente sea una consecuencia de la reforma estatutaria, ni sea objeto que se deba asumir, en el actual estado de la cuestión -o estado del arte, como ahora gustan de decir los anglosajones-, al hilo de esa. Cuando preparaba esas Notas, se me hizo presente un relativamente acalorado debate sobre este asunto con amigos de la infancia, en las navidades de 2005. Que historiadores, ingenieros -agrícolas e industriales- y arquitectos estuvieran interesados en un tema como éste, sólo prueba que su interés trasciende el meramente académico. Al poner en orden mis ideas, no he dejado de pensar en ese animado debate, a sus protagonistas y amigos, José Manuel Macarro, José Manuel y Alfonso Guajardo e Ignacio Camacho, dedico esta reflexión, sobre un asunto al que tanta atención le prestó prontamente el Maestro García-Añoveros, en una enésima prueba de su finura intelectual, de la que tanto seguimos aprendiendo.
} 
ga la profesora Soler Roch, a la que inmediatamente presté mi apoyo incondicional. Originariamente, la idea era dedicar nuestras reflexiones a cuestiones metodológicas, y así se han venido desarrollando, las tres primeras. Para la presente, recibo una cordial invitación a hacerme cargo de su Presentación, del Director del Instituto de Estudios Fiscales, en la que me advierte que se ha preferido no dedicar esta edición de las Jornadas a cuestiones metodológicas, centrando el interés de las mismas en la Reforma de la financiación autonómica en el marco de los nuevos Estatutos.

La primera reflexión que quiero hacer en esta Presentación, es que no estoy precisamente de acuerdo con esa opinión de nuestro querido amigo el Profesor Ruiz-Huertas, aunque desde luego que sí en la necesidad de abordar esta cuestión capital del sistema de financiación autonómica desde una perspectiva técnico-jurídica, como él mismo nos sugiere. Pero, nótese, que esa afirmación ya comporta, una posición metodológica. Con lo que mi primera hipótesis será la de que no es posible afrontar esta cuestión, sino poseyendo un cierto bagaje jurídico, es decir, utilizando los textos normativos fundamentales, la Constitución y los que configuran el bloque de la constitucionalidad, la extensísima Jurisprudencia de nuestro TC y la experiencia de varios modelos de financiación anteriores; sólo así la afrontaremos exentos de prejuicios.

La segunda es reflejo de la enseñanza directa e inmediata de los numerosos trabajos del Profesor García-Añoveros sobre este asunto, que siempre afrontará estas cuestiones jurídicas, con lo que podríamos denominar una visión impura de las mismas ${ }^{2}$, desde la necesidad de hacerlo en el contexto de sus circunstancias políticas, económicas y sociales, en primer lugar. En segundo, apoyándose en el carácter flexible y abierto del modelo constitucional de financiación autonómico, resultado imperativo de la originaria indefinición del modelo de Estado que se concreta en la Constitución ${ }^{3}$. Por último, sin que sea

\footnotetext{
${ }^{2}$ Véase su excelente aportación, de póstuma publicación, en el volumen que recoge las intervenciones en las I Jornada Metodológica Jaime García Añoveros sobre la metodología académica y la enseñanza del Derecho Financiero y Tributario, IEF. Madrid. 2001, bajo el título "El discurso del Método en el ámbito Hacendístico"; de ella, además, dí extensa noticia en mi intervención en esas Jornadas: "Algunas propuestas metodológicas para la (re)construcción de un Derecho Financiero del siglo XXI"

${ }^{3}$ Véase, sobre todo, el arranque del trabajo de Jaime GARCÍA AÑOVEROS "Génesis y desarrollo del modelo de financiación de las Comunidades Autónomas" en AA.VV., Estado regional-Estado federal: La Financiación de las Comunidades Autónomas, Salamanca. Diputación. 1986.
} 
lo menos importante, desde la necesidad de articular un sistema de financiación (tradicionalmente) centralizado en el seno de una organización política (recientemente) descentralizada y, veintiocho años después, progresivamente más descentralizada, mediante la asunción de las fuentes de gasto más importantes de un Estado moderno: la sanidad y la educación.

La última de las categóricas precisiones metodológicas que se imponen para no viciar el debate $a b$ initio, no puede dejar de advertir acerca de lo que podríamos denominar un efecto perverso convergente que se produce en esa dinámica centrípeta y centrífuga, al tiempo. Se trata de constatar una nociva confluencia de intereses entre los dos protagonistas de este asunto, el Estado y las Comunidades Autónomas, de régimen común, que se traduce en una cierta tendencia -tácita, durante mucho tiempo- al mantenimiento del sistema, el primero por el férreo control de los recursos -recuérdese aquéllas afirmaciones de la primera versión de la LOFCA, acerca de la cuasi inmanente imposibilidad de ceder una sola peseta, entonces, del IRPF, so pena de quebrar el Estado, al menos financieramente hablando, si se me permite el barbarismo, por sostener el juego de palabras-; las segundas, por la comodidad de asumir responsabilidades de gasto, sin implicarse en la exigencia de los recursos.

Todas estas circunstancias determinaron el certero diagnóstico del Maestro García-Añoveros, que de forma acabada se plasmaba, tal vez, sin ser conscientes de ello, en el Informe de los Expertos de $1995^{4}$ y que sintetizaba muy bien Esteban QUINTANA ${ }^{5}$ : el modelo de financiación -para ser más precisos, los diferentes modelos aplicados hasta ese momento- se podrían caracterizar por las siguientes notas:

$\checkmark$ escasa autonomía y nula corresponsabilidad fiscal de las CCAA

$\checkmark$ alto endeudamiento de las CCAA, debido básicamente a la rigidez del modelo

\footnotetext{
${ }^{4}$ He manejado la versión publicada en PALAU 14: MONASTERIO ESCUDERO, Carlos; PÉREZ GARCÍA, Francisco; SEVILLA SEGURA, José V.; SOLÉ VILANOVA, Joaquim. Informe sobre el actual sistema de financiación autonómica y sus problemas (Primera parte) 24 (1994); (Segunda Parte) 25 (1995). Información de interés sobre éste puede encontrarse en QUINTANA FERRER, Esteban Reforma de la Hacienda autonómica y Libro Blanco sobre financiación de las Comunidades Autónomas. Valencia, Tirant lo Blanch, 1996.

${ }^{5}$ QUINTANA (1996), 95 ss.
} 
$\sqrt{ }$ ausencia de un explícito criterio de articulación del principio de solidaridad

$\sqrt{ }$ exigua articulación de un principio de coordinación

Enseguida analizaremos, telegráficamente, las grandes fases o etapas de los diversos sistemas de financiación, hasta llegar a la situación que acabamos de sintetizar para pasar, posteriormente, al análisis de la actualmente vigente; antes deberemos recordar, brevísimamente, el marco constitucional en el que debe encajarse este sistema.

\section{EL MARCO CONSTITUCIONAL: LOS PRINCIPIOS INFORMA- DORES Y LOS LÍMITES DEL SISTEMA DE FINANCIACIÓN AU- TONÓMICA. LA POSICIÓN DEL TRIBUNAL CONSTITUCIO- NAL.}

Tal vez, aún cabría suscitar otra cuestión metodológica, si bien ya ha sido mencionada. Nos referimos a la cuestión de la indefinición originaria del modelo de Estado que dimana de la Constitución ${ }^{6}$. Este vuelve a constituir un imperativo categórico del que se deriva la subsiguiente indefinición constitucional del sistema de financiación: sencillamente, no hay tal.

Se formulan principios, se describen límites, se establecen criterios, incluso se declina en la posibilidad de que una Ley Orgánica regule el ejercicio de las competencias financieras de las Comunidades Autónomas, pero la concreción del sistema no está en la Constitución, estará en lo que se vino en denominar el bloque de la constitucionalidad. Y este carácter coyuntural del sistema, no será vicio, sino virtud, como enseguida veremos; pero, en todo caso, así se ha venido produciendo, también acaso, porque en términos macroeconómicos, nadie puede negar esta realidad: la progresiva asunción de competencias por parte de las Comunidades Autónomas inexorablemente incrementa su necesidad de recursos, lo que se suele reconocer, por cierto, con más frecuencia, que su categórico envés, que en la misma medida disminuye la necesidad de los mismos en manos del Estado, con las limitaciones de las que enseguida trataremos.

Principios, límites, criterios para la configuración constitucional del sistema financiero de las Comunidades Autónomas, éste será nuestro inmediato

\footnotetext{
${ }^{6}$ Véanse las pioneras consideraciones sobre este asunto de Álvaro RODRÍGUEZ BEREIJO "Un reflexión sobre el sistema general de la financiación de las Comunidades Autónomas" Revista Española de Derecho Constitucional 15 (1985).
} 
objeto de análisis, mas sin olvidar que éstos no configuran un sistema acaba$\mathrm{do}^{7}$, un marco estricto y cerrado: de ello nos ilustrará con solvencia la Jurisprudencia Constitucional, de la que también nos ocuparemos.

Los tres principios básicos, cimientos de todo el sistema, se encuentran formulados en el art. $157 \mathrm{CE}$ : autonomía financiera, solidaridad (interterritorial) y coordinación. Estos tres principios nucleares, han sido abundantemente desarrollados por la JTC, de modo que en la actualidad no es difícil encontrar una enumeración de principios del siguiente o parecido tenor, en relación con los cuales es posible citar una o varias declaraciones del TC. Nos limitamos a enunciarlos y a señalar la decisión o decisiones del TC que se nos antojan más significativas, en su verificación y aplicación en las distintas fases o existencia de cada uno de los modelos. Mas antes, una consideración de carácter general que debe presidir todo el análisis. Se trata de la necesidad de lograr el necesario equilibrio (dialéctico) entre los principios de autonomía (efectiva) y solidaridad (real). Uno, de carácter centrífugo, de naturaleza centrípeta, el segundo. Y será equilibrio (dialéctico) en cuanto es dinámico y nunca estable, es decir, interdependiente. Se constituirán en una especie de vaso comunicante que al cristalizar en determinados momentos podrán producir el logro de uno a costa o sacrificio (al menos cuantitativamente hablando) del otro. La Jurisprudencia Constitucional he jugado un papel extraordinariamente relevante en la formulación del contenido y alcance de estos principios.

Si partimos de los que se formulan en la LOFCA, y tratamos de analizar su fundamento constitucional, al tiempo que la formulación que de ellos se ha hecho por la JTC, podemos hablar de

$\checkmark \quad$ un principio de neutralidad-lógicamente conectado con el de igualdad y de territorialidad (MARTÍN QUERALT) ${ }^{8}$ - que será la concreción de lo

${ }^{7}$ En esta misma línea ya se habían manifestado RAMALLO MASSANET, Juan y ZORNOZA PÉREZ, Juan "Sistema y modelos de financiación autonómica" en Perspectivas del Sistema Financiero 51 (1995), cuando se fijan como objetivo observar el grado de correspondencia existente entre el sistema de financiación de las CCAA establecido en el bloque de la constitucionalidad y los distintos modelos de financiación aplicados tras la aprobación de la CE (pp. 10).

${ }^{8}$ MARTÍN QUERALT, Juan; LOZANO SERRANO, Carmelo; TEJERIZO LÓPEZ, José M.; CASADO OLLERO, Gabriel. Curso de Derecho Financiero y Tributario. Madrid. Tecnos. 2005; en ese Manual se encuentra la más completa y sistemática exposición sobre los principios y limitaciones del poder financiero de las Comunidades Autónomas, pp. 221 ss.; la exposición que hacemos sigue ese modelo sin demasiadas alteraciones, utilizando las más significativas de las abundantísimas SSTC que se encuentran allí citadas. 
establecido en el art. 139.2 CE y, específicamente, por lo que se refiere a las soluciones tributarias, en el 157.2 CE y su reflejo en el art. 2.1.a) LOFCA: SSTC 37/1981; 8/1986; más recientemente, 96/2002 y 168/2004.

$\sqrt{ }$ un principio de coordinación que tiene su consagración en la actual redacción del art. 2.1.b) LOFCA y trae su fundamento constitucional en el art. 156.1. Encontrará su articulación orgánica en la potenciación de las funciones del Consejo de Política Fiscal y Financiera (CPFF, art. 3 LOFCA) y será la garantía del desarrollo armónico de las diversas Haciendas: SSTC 32/1983; 96/1990 y 62/2001.

$\sqrt{ }$ un principio de solidaridad que se constituye en uno de los pilares del sistema formulado con diversa intensidad y finalidad en los arts. 2, 138.1, 156.1 y $158 \mathrm{CE}$ y concretado en el art. 2.1.c) LOFCA, con carácter general y, en relación con los tributos cedidos, en el art. 19.2: SSTC 25/1981; 135/1992; 64/1990; 109/2004. En cuanto el Estado sale garante del cumplimiento de este principio se establecen instrumentos que lo posibiliten, mediante

- las asignaciones de nivelación (art. 15 LOFCA ex 158.1 CE)

- el Fondo de Compensación Interterritorial (art. 158.2 CE y 16 LOFCA, así como la Ley 22/2001)

$\sqrt{ }$ un principio de suficiencia, que constituirá la encarnadura eficiente de la autonomía [art. 2.1.d) LOFCA], sin aquélla ésta no es sino formulación retórica, como bien puso de relieve el FJ 3 STC 289/2000, al tiempo que caracterizará el modo diferente de cumplirse de acuerdo con el carácter evolutivo de los diferentes modelos de financiación (el denominado paso desde una hacienda de transferencias a un sistema de corresponsabilidad).

$\sqrt{ }$ un principio de lealtad institucional, lo que no es sino exigencia implícita y corolario de todo lo anterior, exigirá que en el Estado de estructura compuesta el ejercicio del poder financiero de los diversos entes territoriales se desarrolle en el ámbito de sus respectivos ámbitos competenciales (MARTÍN QUERALT); se consagra en la nueva redacción que se recoge en el art. 2.1.e) LOFCA; de este principio se puede tener noticias en las SSTC 13/1992, FJ 7 y 48/2004.

Hasta aquí los principios que se recogen el art. 2.1 LOFCA; mas no son 
los únicos, doctrina y jurisprudencia (constitucional) nos permiten formular aún otra serie de ellos, no menos relevantes:

$\sqrt{ }$ un principio de instrumentalidad, cristaliza la idea de que existe una fuerte vinculación que especifica la legitimación de su ejercicio entre la titularidad de la competencia material y la financiera, como ya estableció la muy relevante STC 13/1992. Es principio que debe ponerse en directa relación con el que ya hemos formulado de suficiencia, como hace el FJ 3 STC 289/2000 y, al tiempo, constituirá uno de los límites específicos del ejercicio del poder financiero de las CCAA, las cuales serán competentes en el ámbito y ejercicio de sus competencias, con los límites que esa titularidad les impone (ex art. 156.1 CE, concretado en el art. 1 LOFCA), de ahí que, en aplicación de este principio, ya se haya formulado por la STC 14/1989 (FJ 2) que la potestad de gasto no es título que pueda alterar el orden de competencias diseñado por la CE. Esa vinculación, nos advierte MARTÍN QUERALT, supone otra consecuencia positiva, la autonomía y la suficiencia financiera de las CCAA forman parte del contenido inherente de su ámbito material de competencias y constituye garantía de su autonomía política (con remisión a los FFJ 8 y 3 de las SSTC 135/1992 y 96/2002, respectivamente).

- convendrá recordar, además, que la autonomía financiera es un instrumento indispensable para la consecución de la autonomía política, implica tanto la capacidad de las Comunidades Autónomas para establecer y exigir sus propios tributos como su aptitud para acceder a un sistema adecuado -en términos de suficiencia- de ingresos, de acuerdo con los arts. 133.2 y 157.1 CE (SSTC 179 /1985, 3; 63/1996, 11; 233/1999, 22; 104/2000, 4; 289/2000, 3). Serán los ingresos el soporte material de la autonomía financiera a cuyo fin la LOFCA configurará como principio la suficiencia de los recursos en su art. 2.1.d), según ya hemos señalado supra. Autonomía con relación al ingreso no quiere decir sino capacidad para articular un sistema suficiente de ingresos (FJ 3 STC 289/2000; FJ 4 STC 104/2000).

$\checkmark$ un principio de unidad que está en la esencia de la propia definición de la estructura del Estado, del mismo se pueden encontrar fundamento en los arts. 2, 31.1, 128, 131.1, 138.2 y 139.2 CE; constituirá un límite inherente al derecho a la autonomía (STC 4/1981). La CE garantiza este principio y su convivencia equilibrada con el de la autonomía política de las Comunidades Autónomas, lo que obliga a la búsqueda de constantes equilibrios para la armónica convivencia y respe- 
to de los mismos, pues la unidad de España no es óbice para la coexistencia de una diversidad territorial que admite un importante campo competencial de las Comunidades Autónomas (FJ 11 STC 96/2002). Será el Estado, como veremos de inmediato, el garante de esa unidad, lo que se concretará en una genérica formulación de la igualdad u homogeneidad básica que permita configurar el régimen jurídico de la ordenación de los tributos, por ejemplo, como un verdadero sistema y asegure la unidad del mismo, que es exigencia indeclinable de la igualdad de los españoles (STC 116/1994; en el mismo sentido, 6/1983; 19/1987; 181/1988).

$\sqrt{ }$ un principio de igualdad que encuentra su fundamento en la exigencia de los arts. 138.2 y 139.1 (STC 96/2002). En su aplicación no puede pretenderse que todas las CCAA ostenten las mismas competencias o que deban adornarse de un contenido idéntico. La CE, ha interpretado el TC, no exige un tratamiento jurídico uniforme de los derechos y deberes de los ciudadanos en todo tipo de materias y en todo el territorio del Estado, lo que sería formalmente incompatible con la autonomía, sino, a los sumo, y por lo que al ejercicio de los derechos y al cumplimiento de los deberes constitucionales se refiere, una igualdad de las posiciones jurídicas fundamentales (FJ 10 STC 37/1987; STC 150/1990).

$\sqrt{ }$ un principio de territorialidad específicamente formulado en el art. 157.2 CE, supondrá un (principio) implícito al propio sistema de autonomía territorial (SSTC 13/1988, 101/1995; 132/1996, citadas por MARTÍN QUERALT) y permitirán articular armónicamente la diversidad de las relaciones (financieras) con ocasión del ejercicio de las competencias de las diversas Administraciones Públicas; siendo el territorio el elemento delimitador de las competencias (STC 132/1996), sin que de esta formulación pueda inferirse la absoluta interdicción de que las decisiones (tributarias, por ejemplo) de una Comunidad Autónoma, puedan producir consecuencias fácticas fuera de su territorio (STC 37/1981), criterio reiterado con posterioridad en diversas decisiones del TC $(37 / 1987 ; 150 / 1990 ; 118 / 1996$; 126/2002; 168/2004, todas citadas por MARTíN QUERALT).

- el art. 6 de la LOFCA afronta específicamente esta cuestión al concretarla en relación con el ejercicio del poder tributario propio de las CCAA; en su virtud,

* se establecerá la posibilidad del establecimiento de tributos propios (STC 37/1981; 37/1987; 186/1983; 14/1998; 289/2000) 
* se formulará el principio de coordinación y prohibición de doble imposición con tributos estatales (SSTC 37/1987; 186/1993; 14/1998)

* el mismo principio, si bien reforzado, en relación con los impuestos locales $(37 / 1987 ; 289 / 2000 ; 168 / 2004)$ significa una aplicación específica de la común lealtad constitucional, explicitada en la exigencia de medidas de compensación estatal por ocupación de impuestos autonómicos (art. 6.4 LOFCA).

- por su parte, el art. 9 LOFCA, en relación con los impuestos autonómicos propios, formulará la prohibición de ejercicio del poder tributario con efectos extraterritoriales. El alcance y sentido de este precepto viene matizado por las SSTC 37/1987 y 150/1990, fundamentalmente, lo que se viene a reiterar en el art. 9.b) LOFCA, excluyendo la posibilidad de un gravamen extraterritorial y concretando en el 9.c), lo ya establecido en el 2.1.a) LOFCA, las limitaciones formuladas en los arts 139.2 y $157.2 \mathrm{CE}^{9}$.

Como ya hemos visto, al analizar el principio de unidad, será el Estado el garante de esa unidad, no sólo por lo que se refiere a los aspectos que allí se señalaban respecto de la ordenación de los tributos, como un verdadero sistema, en cuya prosecución será instrumento de primer orden la reserva de ley (STC 19/1987), sino desde una más amplia perspectiva, como recientemente ha puesto de manifiesto, por ejemplo, la STC 3/2003 que, al resumir el papel fundamental de las Cortes Generales, al tiempo que las relaciones entre los arts. 31.3 y 133, nos advierte en su FJ 4: las Cortes Generales ejercen, como hemos dicho, una función específica y constitucionalmente definida a la que hicimos referencia en la STC 76/1992, de 14 de mayo [FJ 4 a)]. A través de ella, cumplen tres objetivos especialmente relevantes: a) Aseguran, en primer lugar, el control democrático del conjunto de la actividad financiera pública (arts. 9.1 y 66.2, ambos de la Constitución); b) Participan, en segundo lugar, de la actividad de dirección política al aprobar o rechazar el programa político, económico y social que ha propuesto el Gobierno y que los

\footnotetext{
${ }^{9}$ Véanse las interesantísimas páginas de RUIZ ALMENDRAL, Violeta Impuestos cedidos y corresponsabilidad fiscal. Valencia. Tirant lo Blanch. 2004, sobre la STC 150/1990 y la posibilidad de entender el alcance de la prohibición de extraterritorialidad del art. 9 LOFCA puesto en relación con el art. 157.2 CE (pp. 367 ss). También pondrá en conexión la corresponsabilidad fiscal con la nueva configuración de los impuestos cedidos Me Desamparados MORA LORENTE Impuestos Cedidos: implicaciones internas y comunitarias. Valencia. Tirant lo Blanch. 2004.
} 
presupuestos representan; c) Controlan, en tercer lugar, que la asignación de los recursos públicos se efectúe, como exige expresamente el art. $31.2 \mathrm{CE}$, de una forma equitativa, pues el presupuesto es, a la vez, requisito esencial y limite para el funcionamiento de la Administración (FJ 4 STC 3/2003).

Será competencia del legislador estatal la regulación de las instituciones comunes a las distintas Haciendas y la fijación del común denominador normativo que garantice las condiciones básicas de cumplimiento del deber de contribuir: la indudable conexión existente entre los arts. 133.1, 149.1.14ํㅡ y 157.3 determina que el Estado sea competente para regular no sólo sus propios tributos, sino también el marco general de todo el sistema tributario y la delimitación de las competencias financieras de las Comunidades Autónomas respecto de las del propio Estado (FJ 6 STC 192/2000). En esa búsqueda de un mínimo de uniformidad, en beneficio de la igualdad básica de todos los españoles en el campo económico y financiero, se ha manifestado en diversas ocasiones el TC a favor de atribuir ese cometido al Estado (SSTC 1/1982; 52/1988, FJ 3), al tiempo que subraya su papel de garante de la denominada unidad de mercado (SSTC 88/1986, FJ 6; 64/1990, FJ 3). A estas ideas parece responder el art. 1 LGT cuando establece su aplicación a todas las Administraciones tributarias en virtud y con el alcance que se deriva del art. 149.1.1 $1^{\underline{a}}, 8^{\underline{a}}, 14^{\underline{a}}$ y $18^{\underline{a}} \mathrm{CE}$, en la medida en que en la LGT se establecen los principios y las reglas juridicas generales del sistema tributario español.

Como nos acaba de advertir la STC 192/2000, es necesario poner en relación los arts. 133.1 y 149.1.14ª . Será por tanto el Estado, como titular de esa competencia, quien pueda concretar mediante ley orgánica (ex 157.3 CE) el sistema de distribución de competencias financieras y tributarias a partir de principios presentes en la CE como son, según acabamos de ver, básicamente, los de autonomía y solidaridad, presididos por el de coordinación: la posibilidad de regulación mediante LO que establece el art. 157.3 viene así entendida por el TC: ... no se pretendió sino habilitar la intervención unilateral del Estado en ese ámbito competencial a fin de alcanzar un minimo grado de homogeneidad en el sistema de financiación autonómico (FJ 9 STC 68/1996); como el FJ 10 de la STC 68/1996 expresamente reconoce, el sistema LOFCA no era sino uno de los posibles, ya lo hemos dicho, y convendrá recordarlo más adelante, cuando desde voces políticamente interesadas se desacredita toda modificación, sin pensar que entre 1996 y 2004 se ha producido una de las reformas más atrevidas de la LOFCA, como la que experimenta en virtud de la LO 7/2001 y, prácticamente, generaliza el sistema de cesión de tributos, predicándolo respecto de algunos que hasta la fecha, tal parecían que eran ontológicamente estatales. 
Intentando sintetizar los aspectos más señalados de este rol que al Estado se le atribuye podemos fijar nuestra atención en los siguientes:

$\sqrt{ }$ será el titular del poder tributario para establecer el sistema tributario estatal, asi como el marco general de todo el sistema tributario: arts. 31.1 CE y 133.1 CE. En este sentido, convendrá recordar la idea de sistema como una de las funciones sustantivas atribuidas al principio de reserva de ley e indirectamente a su titular, el Estado mediante las Cortes Generales (STC 19/1987); idea que reiterará la STC 116/1994: idea de sistema tributario presidido por un conjunto de principios generales susceptibles de garantizar la homogeneidad básica, asegurando un cierto grado de unidad, que es exigencia indeclinable de la igualdad de los españoles. Como ha dicho el profesor MARTÍN QUERALT, será doctrina del TC que es competencia del legislador estatal la regulación de las instituciones comunes a las distintas Haciendas y la fijación del común denominador normativo que garantice las condiciones básicas de cumplimiento del deber de contribuir, véase en este sentido el FJ 6 STC 192/2000.

* La idea básica será que sólo el Estado es el garante de la unidad de la Nación española (art. $2 \mathrm{CE}$ ); de la unicidad del orden económico nacional, presupuesto necesario de la propia existencia del Estado de las Autonomías (STC 1/1982), así como de la igualdad básica de todo los españoles en la totalidad del territorio nacional (SSTC 53/1988, FJ 3), condición para que pueda producirse la unidad de mercado (STC 88/1986, FJ 6; 64/1990, FJ 3). De esta función se hará eco el art. 1 LGT, como ya vimos.

$\checkmark$ será el titular de la competencia para el establecimiento de los criterios básicos informadores del sistema tributario de las CCAA. De nuevo habrá que acudir a los arts. 157.3 CE y a sus resortes fundamentadores o legitimadores: los arts. 133.1 y 149.1.14ª: es función del legislador estatal concretar el sistema de competencias financieras y tributarias entre el Estado y las CCAA. Su concreción es la LOFCA (en sus diversas versiones); véase esta idea en el FJ 9 STC 68/1996. De ahí que, como acabamos de ver, sea la LOFCA la norma jurídica fundamental para analizar las pautas y límites de configuración del sistema financiero de las CCAA. No obstante convendrá, insistir en que la LOFCA no es sino una posible concreción de ese sistema financiero; ese carácter evolutivo del sistema no ha pasado desapercibido a la JTC: véase, por ejemplo, STC 68/1996, FJ 10; 289/2000, FJ 3; 168/2004, FJ 4. Sobre esta cuestión habremos de volver posteriormente. 
Para cerrar este epígrafe, sólo nos resta fijar nuestra atención en otra de las cuestiones constitucionalmente abiertas, en relación con el sistema de financiación de las Comunidades Autónomas. Se trata de poner de manifiesto el carácter abierto de la relación de recursos contenida en el art. 157 CE, que se limitará a su enumeración, sin fijación del rol relativo de cada uno de ellos. Lo que significará no sólo dejar abierto el sistema, sino incluso el modelo.

No será inocuo observar, a estos efectos, cómo cada uno de los modelos se ha caracterizado por ir modificando ese rol, sobre todo si atendemos al aspecto cuantitativo de su relevancia en el conjunto del sistema, como ya vimos que ponía de relieve, por ejemplo, el FJ 3 STC $289 / 2000^{10}$, hasta el punto de constituirse en el referente identificador de cada uno de ellos.

Si bien no siempre, en algunas ocasiones, sí se han producido, al hilo de las modificaciones del modelo, cambios o alteraciones en parte del marco nor-

\footnotetext{
${ }^{10}$ No obstante la extensión del pasaje, merece la pena ser reproducido en su totalidad: Es cierto que la autonomía financiera ha venido configurándose desde sus orígenes más por relación a la vertiente del gasto, que con relación al ingreso — como capacidad para articular un sistema suficiente de ingresos(SSTC 13/1992, de 6 de febrero, FJ 7, y 104/2000, de 13 de abril, FJ 4); por ello, es evidente que el sistema de financiación de las Comunidades Autónomas se ha venido articulando desde la óptica de unas Haciendas territoriales de transferencia en las que el grueso de sus ingresos procedian del Presupuesto estatal, a través del porcentaje de participación en los ingresos del Estado (SSTC 13/1992, de 6 de febrero, FJ 7, y 68/1996, de 18 de abril, FJ 10). No obstante, no cabe duda alguna de que en los últimos años se ha pasado de una concepción del sistema de financiación autonómica como algo pendiente o subordinado a los Presupuestos Generales del Estado, a una concepción del sistema presidida por el principio de "corresponsabilidad fiscal" y conectada, no sólo con la participación en los ingresos del Estado, sino también y de forma fundamental, de la capacidad del sistema tributario para generar un sistema propio de recursos como fuente principal de los ingresos de Derecho público. Basta con acudir al último modelo de financiación, correspondiente al quinquenio 1997-2001 (con la consiguiente modificación de la Ley Orgánica 8/1980, de 22 de septiembre, de Financiación de las Comunidades Autónomas, por la Ley Orgánica 3/1996, de 27 de diciembre; y con la aprobación de la nueva Ley 14/1996, de 30 de diciembre, de Cesión de Tributos del Estado a las Comunidades Autónomas), para comprobar cómo se ha puesto de manifiesto la voluntad del legislador estatal de estructurar un nuevo sistema de financiación menos dependiente de las transferencias estatales y más condicionado a una nueva estructura del sistema tributario que haga a las Comunidades Autónomas "corresponsables" del mismo (dicha intención y a venía manifestada en los "Acuerdos para el desarrollo del sistema de financiación de las Comunidades Autónomas del quinquenio 1992-1996”, aprobados por el Consejo de Política Fiscal y Financiera el día 7 de octubre de 1993). Concepto éste el de la "corresponsabilidad fiscal", que no sólo constituye la idea fundamental de dicho modelo sino que además se erige en el objetivo a conseguir en los futuros modelos de financiación. En este sentido se ha manifestado la Exposición de Motivos de la Ley 14/1996 citada donde se proclama que "a partir de estos momentos se inicia una nueva etapa en la evolución histórica del régimen de cesión de tributos del Estado a las Comunidades Autónomas, presidida por el principio de corresponsabilidad fiscal efectiva".
} 
mativo general: la LOFCA y la Ley de Cesión, por ejemplo, fueron objeto de modificaciones, tanto en 1996 como en 2001, fechas, precisamente, del penúltimo y antepenúltimo de los modelos de financiación, mas ello no implicaba, anótese con claridad, una modificación ni de los Estatutos ni del marco constitucional, por supuesto, como ya veíamos, con carácter general. Esa falta de fijación constitucional del rol relativo de cada uno de los recursos, a su vez, significó que una alteración tan relevante como se produce en el concepto de tributo cedido - por su extensión e intensidad- en la plasmación de los dos últimos modelos, sólo tuvo que reflejarse en la LOFCA -si bien con notables defectos técnicos, como por ejemplo, en los que incurre el art. 10 LOFCA (en su versión de 2001), al no mencionar como nota caracterizadora del tributo cedido que aparece en su art. 10, el de la eventual atribución de la gestión a la Comunidad Autónoma- y, lógicamente, en la Ley de cesión, la 21/2001.

\section{LOS DIVERSOS MODELOS DE FINANCIACIÓN: DE LA HA- CIENDA DE TRANSFERENCIAS AL SISTEMA DE CORRESPON- SABILIDAD.}

Un aspecto metodológico de especial interés, a los fines para los que somos convocados, consistirá en subrayar cómo todas las soluciones LOFCA han sido posibles con un mismo texto constitucional y con un mismo texto estatutario, como acabamos de señalar. Desde esta premisa se nos antoja que pudiéramos preguntarnos ¿pudiera ello significar, entonces, que el binomio causa efecto que parece inferirse del lema de estas Jornadas, no sea sino pura contingencia, no fenómeno inmanente a la propia modificación estatutaria? $\mathrm{O}$, tal vez, sin confesarlo abierta e inmediatamente, ¿la ola reformista de los Estatutos, es el resultado, entre otras causas, de la insatisfactoria situación financiera a que han conducido las sucesivas reformas de los sistemas de financiación?

Si conjugamos ambos aspectos, pudiera plantearse la cuestión, a su vez, de este otro modo, seguramente la respuesta a la primera cuestión sea afirmativa, otra cosa es que esas modificaciones estatutarias pudieran dar lugar, efectivamente, a una sustantiva alteración del modelo de financiación, lo que nos proporcionaría una respuesta negativa. La paradoja estribaría entonces a que el sí, o al menos, el sí, seguramente, entre otras causas, no puede determinar sino un no a la segunda de las cuestiones. Y tal vez deberíamos discutir, precisamente, sobre los anclajes jurídicos de la proposición que acabo de hacer, máxime cuando se ha debatido en términos no muy sólidos, intelectualmente hablando, de un desterrado sistema de bilateralidad, que desde luego no propugno, aunque dejo encima de la mesa. 
Un breve repaso a los diversos modelos de financiación ${ }^{11}$, con señalamiento de sus pautas caracterizadoras, nos proporciona las siguientes conclusiones:

$\sqrt{ }$ una primera etapa que podríamos concretar en tres grandes fases caracterizada esencialmente por lo que ha venido en denominarse [incluso por la JTC -STC 289/2000, FJ 3 (ver la nota 10)-] una hacienda de transferencia y que engloba

- un período transitorio (1980-1986): durante esta etapa se garantiza por el Estado, mediante un porcentaje de participación en los impuestos estatales no cedidos y la recaudación de los cedidos, la financiación de los servicios transferidos, con una cantidad igual al coste efectivo del servicio en el momento de la transferencia (QUINTANA FERRER) ${ }^{12}$; de este coste efectivo se excluyen las subvenciones que llevaban aparejadas los servicios traspasados y los recursos necesarios para financiar la inversión nueva. Uno de los efectos perversos del sistema es que se produce un recurso masivo al endeudamiento, prueba de la insuficiencia del sistema.

- un período definitivo (sic, 1987-1991): se caracteriza por el abandono del sistema de cálculo de los porcentajes de participación basados en el coste efectivo de los servicios en beneficio de otro en el que operaban una serie de variables socioeconómicas plasmadas en el art. 13 de la LOFCA entonces vigente. Se dota al sistema de participación de mayor objetividad y automatismo. Se amplió el alcance de los tributos cedidos, sin embargo, la derogación del Impuesto de Lujo por la entrada en vigor del IVA supuso una notable disminución de los ingresos fiscales cedidos a las CCAA (MONASTERIO ESCUDERO) $)^{13}$, lo que determinó, que se incrementara la parte del coste efectivo a financiar a través de la PIE, aunque dotándose a este instrumento de financiación incondicionada de mayor estabilidad al pasar a aplicarse sobre los Ingresos Tributarios Ajustados Estructuralmente (MONASTERIO ESCUDERO), amén de lo

${ }^{11}$ Un excelente análisis de los diferentes modelos puede encontrarse en RAMALLO/ZORNOZA (1995) PP. 13 a 30.

${ }^{12}$ QUINTANA (1996), 26-27.

13 MONASTERIO ESCUDERO, Carlos. "El sistema de financiación autonómica 2002" en SALINAS JIMÉNEZ, Javier (dir.) El nuevo modelo de financiación autonómica (2002). Madrid. IEF. 2002. 
que supuso de aumento absoluto bruto de esta base de aplicación. Se coordina el FCI con los fondos europeos (por ejemplo el FEDER), sustrayendo de los mismos la parte destinada a financiar la inversión nueva de los servicios de las CCAA (QUINTANA FERRER) ${ }^{14}$.

- un período de tímido inicio de la denominada corresponsabilidad fiscal (1992-1996) caracterizado por la articulación de una serie de nuevas medidas: una participación territorializada en el 15\% de las cuotas líquidas del IRPF; se instauran una serie de reglas de coordinación en materia de endeudamiento, mediante la aplicación del denominado Escenario de Consolidación Presupuestaria (ECP). No obstante, se continúa confirmando la importancia de las transferencias de la PIE, como el eje central del sistema de financiación; la masa global de recursos a cargo de la PIE vuelve a aumentar y aunque se produce algún cambio en las ponderaciones de las variables de reparto, en líneas generales se mantiene la continuidad en el sistema de transferencias incondicionadas (MONASTERIO ESCUDE$\mathrm{RO})^{15}$.

- el resultado de estas tres fases es un incremento paulatino de la dependencia financiera de las CCAA de régimen común, por lo que pasa a ocupar un primerísimo plano el debate sobre la corresponsabilidad fiscal, cuestión que centra el Acuerdo del CPFF de diciembre de 1996 y que supuso un primer paso sustantivo en esa dirección.

$\checkmark$ una segunda etapa puede venir a ser caracterizada, como ya lo hizo la STC 289/2000, por constituir o tender a terminar siendo un modelo presidido por el principio de corresponsabilidad fiscal y conectada, no sólo con la participación en los ingresos del Estado, sino también y de forma fundamental, con la capacidad del sistema tributario para generar un sistema propio de recursos como fuente principal de los ingresos de derecho público; es decir, un sistema menos dependiente de las transferencias estatales y más condicionado a una nueva estructura del sistema tributario que haga a las CCAA corresponsables del mismo.

\footnotetext{
${ }^{14}$ QUINTANA (1996), 28.

${ }^{15}$ MONASTERIO (2002), 18.
} 
- el debate acerca de la corresponsabilidad fiscal y las soluciones que mejor caracterizan el modelo del quinquenio 1997-2001 es lograr un reducción de la importancia relativa de las transferencias como recurso de las CCAA. Ello se intenta lograr con un notable incremento de la cesión del porcentaje de participación en el IRPF, cuyo límite se fija en el 30\% -lo que supuso una modificación de la LOFCA, para incluir al IRPF entre los cesibles- y con una solución, muy celebrada en su momento, cual fue la cesión de específicas competencias normativas en relación con los tributos cedidos.

* en la práctica ha dado lugar a perversiones importantes en el sistema con un ejercicio de esa competencia no precisamente adornada de un principio de lealtad institucional horizontal, es decir, entre las mismas CCAA. Mas sobre todo porque, desde luego, su ejercicio no ha ido, en ningún caso, en la línea de incrementar notablemente el índice de participación de estos recursos en el montante global de la recaudación propia -que no otro era el objetivo de este incremento del alcance potencial de la cesión-, sino, más bien, me aventuraría a decir, que todo lo contrario. Una hipótesis -no sé si metodológica- me atrevo a formular: con el ejercicio de las competencias normativas en relación con los tributos cedidos ninguna Comunidad Autónoma ha incrementado en términos relativos su recaudación, es decir, que el ejercicio de la corresponsabilidad fiscal, en la práctica está todavía muy lejos de proporcionar los objetivos que se le señalaban en el Acuerdo.

$\checkmark$ las causas del fracaso de la cesión de competencias normativas para el logro de los objetivos de reducir el peso específico de las transferencias son variadas y no quedan -ni siquiera metodológicamente- bajo la competencia de juristas: un acertado análisis de las mismas puede verse en el trabajo de MONASTERIO ESCUDERO que venimos citando, donde además se citan causas diferentes a tenor de circunstancias diversas que procuraban que el sistema tuviera más o menos interés según respecto de qué Comunidad Autónoma se tratara, paradoja que proporcionaba, además, un desincentivo a asumir ese tipo de competencia.

$\checkmark$ el nuevo modelo de financiación en vigor desde 2002 es el resultado de los Acuerdos del CPFF de 27 de julio, de 16 y 22 de noviembre de 2001. Desde luego su primera gran virtud es que se plasman en nor- 
mas jurídicas que pueden superar fácilmente el reproche del déficit democrático que algunos han imputados a los acuerdos adoptados en el seno de ese órgano, por cuanto ahora son normas jurídicas con rango de ley -orgánica, en el caso de la LOFCA-que se aprueban en las Cortes. Esos acuerdos supusieron, en lo esencial, la necesidad de una nueva redacción de algunos preceptos de la LOFCA, lo que se hace en virtud de lo dispuesto en la LO 7/2001; de una nueva Ley de Cesión, que se concreta en la Ley 21/2001; la Ley 22/2001, en relación con la nueva regulación del FCI y, finalmente, en aplicación del pacto de estabilidad presupuestaria, la LO 5/2001. La seguridad jurídica, es desde luego, un principio que ahora se satisface de modo más completo con esa técnica.

- de cualquier modo, no es posible dejar de señalar cómo, al tiempo que el nuevo modelo sigue sin exigir una alteración de los Estatutos o del marco constitucional, sí es preciso, esta vez, una modificación -intensa, me atrevería a decir-, de la norma que preveía el 157.3 CE, es decir, la LOFCA y la Ley de Cesión; en consecuencia, de todas las nuevas leyes de cesión que se promulgaron el 1 de julio de 2002 (BOE de 2 de julio). Y ello porque, en mi opinión, los Acuerdos de 2001 suponen un paso de gran relevancia en la búsqueda de los objetivos que se habían marcado con el modelo anterior. Sobre todo, en la búsqueda de hacer frente a los dos lunares de todo el proceso de financiación autonómica: un grado efectivo de corresponsabilidad fiscal; un más acentuado cumplimiento del principio de suficiencia, sin dependencia tan intensa de las transferencias, como nota caracterizadora del sistema. ¿Cuáles son esos instrumentos?

$\checkmark$ en primer lugar, se propugna un modelo integrado de financiación, es decir, ya no se distinguirá entre competencias comunes y educativas, de una parte, y sanidad de otra; ello es un avance significativamente positivo en la medida en que desaparecen las transferencias específicas para financiar la sanidad que pasan a integrarse en un Fondo de Suficiencia, convertido en el sistema general de transferencias incondicionadas $^{16}$, para la validez de esta caracterización general del sistema, no debe ser óbice que se haya fijado una cantidad mínima a destinar a la financiación sanitaria, debido, sobre todo, al fuerte incremento

\footnotetext{
${ }^{16}$ Como sostiene MONASTERIO (2002), este fondo será el continuador tanto de las trasferencias específicas sanitarias, como de las transferencias de la PIE.
} 
de este gasto, lo que pronto dejará de ser, al menos formalmente, un elemento de restricción que desmerezca la generalización del sistema que hemos apreciado.

$\sqrt{ }$ en segundo lugar, esa generalidad es virtud, asimismo predicable del sistema en cuanto es asumido por la totalidad de las CCAA de régimen común.

$\sqrt{ }$ en tercer lugar, su duración es indefinida, lo que aporta estabilidad al sistema. Ahora se plantea una modificación del modelo, como consecuencia ( $s i c$ ) de los nuevos Estatutos, lo que no afecta a esta vocacional caracterización de constituirse potencialmente en un modelo esta$b l e$, es decir, no nato con una temporalidad predeterminada. De ello, como es sabido, se hará eco, el art. 13 LOFCA.

$\sqrt{ }$ en cuarto lugar, se produce una ampliación de la cesta de tributos cedidos (IVA, IIEE, aunque en porcentajes diversos) y, en algunos casos, de los porcentajes de cesión y con algunas cesiones nuevas, asimismo, como en el caso de los impuestos sobre la electricidad y determinados medios de transporte. En otros, se amplían las competencias normativas.

$\sqrt{ }$ otra notable novedad es la reiterada posibilidad de ejercer la corresponsabilidad fiscal a través de los recargos. Se podrán ejercer sobre todos los tributos susceptibles de cesión, con excepción del Impuesto sobre Ventas Minoristas de Hidrocarburos. En relación con el IVA y los IIEE, en tanto no se tengan competencias normativas sobre los tipos no podrán establecer recargos; sí se podrán establecer en relación con el impuesto especial de matriculación. Esta solución no parece demasiado razonable, desde la perspectiva de que en relación con los tributos susceptibles de cesión, que son los cedidos efectivamente, ya se tienen competencias normativas -plenas o limitadas-, en relación con los tipos; en aquellos supuestos en que no se tienen, tampoco se ha cedido la competencia normativa en materia de tipos (art. 12 LOFCA), de ahí que la doctrina, por ejemplo MONASTERIO ESCUDERO, sostenga que se produce una incoherencia entre ambas posibilidades, ya que elevando el tipo se consigue el mismo objetivo que estableciéndolo más bajo y complementándolo con un recargo. En su opinión ello conduce a la marginalidad de este tipo de recursos.

$\checkmark$ para terminar con este breve repaso al modelo actualmente vigente, convendrá hacer referencia a dos cuestiones especialmente contro- 
vertidas hasta ahora: nos referimos al sistema de transferencias y a la posibilidad y modo de endeudamiento de las Comunidad Autónomas.

$\checkmark$ en relación con las transferencias habrá que distinguir entre las condicionadas y las incondicionadas. De suma importancia es ésta concretada en el denominado Fondo de Suficiencia (FS). Entre las condicionadas deberemos estudiar las denominadas Asignaciones de Nivelación de los Servicios Públicos Esenciales (ANSPE) y el Fondo de Compensación Interterritorial (FCI).

- el FS supone la concreción de una generalización de las transferencias que ya vimos con anterioridad; las cuestiones fundamentales son el modo en que se calcula su cuantía y las reglas para su evolución futura. A partir del volumen global de recursos a distribuir, tomando como referencia los valores del año base 1999, se calculará aquélla. Los recursos se repartirán de acuerdo con una serie de ponderaciones para hacer efectivo el principio de solidaridad. Dentro de esa generalización de la transferencia, se establecen tres bloques diferenciando entre competencias comunes y educativas (GCE); sanitarias (GSA) y competencias de servicios sociales (GSS). Los criterios de ponderación serán diferentes para cada bloque; así, por ejemplo, el padrón de población (94\%) ponderará el bloque de competencias comunes y educativas, al tiempo que la superficie en $\mathrm{Km}^{2}(4,2 \%)$ o la insularidad $(0.6 \%)$, mientras que en el caso del bloque de competencias sanitarias, los elementos de ponderación serán la población protegida $(75 \%)$, la insularidad $(0.5 \%)$ o, en el bloque de las competencias de servicios sociales, la población superior a 65 años $(100 \%)$, será el único elemento de ponderación. La segunda variable para determinar la parte correspondiente a cada Comunidad del FS, serán los recursos a distribuir en cada bloque, en el primer caso, por ejemplo, la PIE, el Fondo de garantía para 1999 o los tributos cedidos y tasas transferidas; en el segundo, la financiación sanitaria de las CCAA con sanidad asumida ${ }^{17}$. A esta distribución de recursos hay que añadir un fijo mínimo para cada Comunidad de 39,66 millones de euros. El sistema específico de explicitación de la parte de FS para cada CCAA es el resultado de un complejo cálculo ${ }^{18}$.

\footnotetext{
${ }^{17}$ Datos tomados todos del trabajo que citamos de MONASTERIO (2002).

${ }^{18}$ Consúltese por los interesados MONASTERIO (2002), 44 ss.
} 
- novedad noticiable será que por vez primera se establecen los criterios para fijar lo que son servicios públicos fundamentales (art. 15.1 LOFCA), así como los supuestos en los que se deben considerar afectados los niveles de prestación de los servicios públicos esenciales (art. 67 Ley de Cesión). Se consideran servicios públicos esenciales los de educación y sanidad. Tanto en educación, como en sanidad, se considerarán afectados cuando el incremento de alumnos en los niveles de enseñanza obligatoria supere en tres puntos la tasa media de incremento nacional; en el supuesto de la sanidad, cuando el incremento de la población protegida -ponderada por la edad-supere asimismo los tres puntos respecto de la media nacional. Teniendo en cuenta la superficie, si la Comunidad Autónoma es superior a $90.000 \mathrm{Km}^{2}$, será suficiente el incremento de un punto porcentual. Dadas estas circunstancias, la asignación no es necesariamente automática, y sería causa de una revisión del FS, si se reiterasen en un plazo inferior a cinco años.

- por lo que se refiere al FCI, se trata, como ya dijimos, de una modalidad de transferencia condicionada. Su regulación encuentra cabida en la Ley 22/2001, ahora se afectan una parte de estos recursos a gastos de inversión, pudiendo destinarse un $25 \%$ a los gastos corrientes derivados de aquellos.

$\sqrt{ }$ en relación con el endeudamiento, hay que tener en cuenta que el diseño originario de la LOFCA, plasmado en el art. 14, se ve afectado en gran medida por la LO 5/2001 complementaria de la LGEP. Ahora a los requisitos de ser destinados los recursos así obtenidos exclusivamente a gastos de inversión y que el importe total de las anualidades de amortización no excedan del $25 \%$ de los gastos corrientes de la CCAA, habrá que añadir una específica autorización del Estado que habrá de tener en cuenta el cumplimiento del principio de estabilidad presupuestaria del art. 2.1.b) LOFCA. Adquieren una mayor transparencia estas decisiones, por cuanto se adoptarán en el seno del CPFF coordinándose entre sí y con las del Estado (art. 14.4 LOFCA).

\section{LA REFORMA DE LA FINANCIACIÓN AUTONÓMICA CON OCASIÓN DE LOS NUEVOS ESTATUTOS DE AUTONOMÍA.}

La existencia de los nuevos Estatutos no determina efectiva e inexorablemente la necesidad de arbitrar un nuevo sistema de financiación, ésta es una conclusión jurídicamente cierta, aunque política y económicamente, más que 
discutible; y ambos planos deben ser tenidos en cuenta, pues si los dos aspectos terminales de la segunda afirmación fueren ciertos, sería necesario alterar la primera: la inexorabilidad, no será jurídica, pero se impondría y su carácter de imperativo categórico la haría inexorable. Aunque jurídicamente no fuere imprescindible, el diseño de un nuevo modelo, que no podría predeterminar el texto estatutario, y el insatisfactorio resultado del existente, determinarían -de hecho- esa efectiva relación de causa -que no de efecto- y resultado: se convertiría en inexorable la necesidad de diseñar un nuevo modelo de financiación.

Un breve repaso a las líneas maestras en materia de financiación de los tres Estatutos que más han avanzado en el proceso de su Reforma (Valencia, Cataluña y Andalucía), nos permitirán afianzar, con fundamentos jurídicos, estas afirmaciones que tienen una indudable vocación metodológica, como corresponde al objeto difuso de estas Jornadas.

Una segunda consideración general, nos llevaría de la mano del Prof. Fernando PÉREZ ROYO a la siguiente conclusión: el art. 133.2, leído tras el proceso de desarrollo del Título VIII de la CE, en cuya virtud todas las CCAA tienen atribuida competencia legislativa, no puede sino entenderse en el sentido de que éstas, no están en paridad con las Corporaciones Locales, no serán, por tanto, limitaciones de similar índole, las que regulen y condicionen el ejercicio de sus competencias financieras; dicho de otra manera, y con palabras del profesor sevillano, el auténtico mandato del precepto sería el siguiente: Las CCAA pueden establecer y exigir tributos, de acuerdo con la Constitución y su Estatuto y de conformidad con lo que en su caso, establezca la Ley Orgánica del art. 157.319.

Si seguimos el proceso intelectivo del profesor de la Universidad de Sevilla, se suscitaría el debate, que en un momento determinado, ha sido central, acerca de si es constitucionalmente viable que un Estatuto, por ejemplo, el ca-

19 PÉREZ ROYO, Fernando. "Las reformas estatutarias y el modelo de financiación" (en prensa); su sutil apreciación no es más que el reflejo de la cuestión que apreciábamos en el inicio de estas consideraciones: indefinición del modelo de Estado de las Autonomías y su consiguiente indeterminación de las competencias financieras, es decir, de su sistema de financiación, aspecto que necesariamente comporta el de sus competencias. Teniendo en cuenta que este trabajo se redacta durante el invierno de 2006, es aún más apreciable su coincidencia intelectual con lo que definitivamente se llega a formular en el art. 201.1 del Estatuto de autonomía de Cataluña: Las relaciones de orden tributario y financiero entre el Estado y la Generalitat se regulan por la Constitución, el presente Estatuto y la Ley Orgánica prevista en el apartado tercero del art. 157 de la Constitución. 
talán, diseñara su propio y específico sistema de financiación. Y ahora entramos de lleno a tratar del lema de estas Jornadas, aunque algunos pueden pensar -Ya era hora...!- en fin, ésta es la cuestión con que queremos cerrar ésta ya demasiado larga Presentación, que no sé si los organizadores, en este momento, lamentarán haberme encargado.

Para responder a estas cuestiones un elemento importante a tener en cuenta es el carácter no imperativo de la LOFCA, es decir, ésta no tiene, según el art. $157.3 \mathrm{CE}$, carácter necesario, sino meramente facultativo, como tantas veces, a lo largo de mis palabras, he reiterado, porque lo considero afirmación nuclear del debate genérico suscitado, y, desde luego, del específico que proporciona el lema de estas Jornadas, aunque en el actual estado de desarrollo del sistema y con la JTC, la conclusión, tal vez, sea muy distinta, como enseguida se verá.

En segundo lugar, cuando se han planteado conflictos entre la LOFCA y preceptos concretos de algún Estatuto, la JTC se ha inclinado, en opinión de Fernando PÉREZ ROYO, por subrayar el papel cardinal de la LOFCA, entendiendo el contenido del art. $157.3 \mathrm{CE}$, como un precepto que tiene atribuida la función primordial de asegurar cierto grado de uniformidad en la financiación autonómica ${ }^{20}$. La JTC ha puesto el acento en la finalidad de la norma estatal: alcanzar un mínimo grado de homogeneidad en el sistema de financiación autonómica; su función no es sino la de permitir que una LO pudiese insertarse en el bloque de la constitucionalidad delimitador del concreto alcance de las competencias autonómicas en materia financiera (FJ 9 STC 68/1996; STC 181/1988, FJ 8; 183/1988, FJ 3; 250/1988, FJ 1; 150/1990, FJ 3).

Si bien la LOFCA es una norma contingente, pudiera plantearse el supuesto del establecimiento de un régimen particularizado en un Estatuto que supusiera una parcial derogación de la LOFCA; el Prof. Fernando PÉREZ ROYO no desdeña esta posibilidad y considera que la Constitución no la prohíbe, aunque la limita, al exigir que ese sistema tributario no puede entrañar privilegios económicos, ni prever otros recursos que los expresamente mencionados en el art. 157.1 CE; por último, afirmará que el régimen de Concierto que directamente se prevé en la DA Primera CE, es específico y propio de la Comunidad Autónoma ahí contemplada y, por tanto, un régimen financiero específico, de otra que no fuera la ahí contemplada, no podría aspirar a tener

${ }^{20}$ PÉREZ ROYO (2006). 
un régimen de concierto, sino, insistimos, un régimen basado en las fuentes señaladas en el art. 157.1, que no necesariamente respondiera a pautas generales.

Yo creo que este planteamiento del prof. Fernando PÉREZ ROYO es interesante y podríamos empezar a hablar de este aspecto de la cuestión en los debates posteriores a lo largo del día de hoy. Yo creo que es una solución que plantea serias dudas, una vez que se ha convertido la LOFCA, no ya en una mera posibilidad, como preveía la CE, sino en una realidad insertada en el bloque de la constitucionalidad y que, según acabamos de ver, tiene atribuida a tenor de la JTC la función de [delimitar] el concreto alcance de las competencias autonómicas en materia financiera (FJ 9 STC 68/1996, por todas).

Junto a ese argumento jurídico, habría otro, tal vez más contundente: la admisibilidad de esa posibilidad que se enfrentaría frontalmente con el mínimo grado de homogeneidad en el sistema de financiación autonómico, a que hacía referencia la JTC, produciría una especie de carrera generalizada y emulatoria de algunas CCAA -las de mayor capacidad económica-, en beneficio de su autonomía financiera, mas, al tiempo, en perjuicio de las de menor capacidad, en manifiesto detrimento del principio de solidaridad. Un mínimo de homogeneidad es inevitable para impedir que ese fenómeno que acabo de señalar pudiera producirse; de todos modos, es cuestión que podríamos debatir y sobe la que no poseo una opinión absolutamente definida, aunque me inclino por negar la viabilidad, incluso jurídica, de esa posibilidad.

Finalmente, se ha suscitado la posibilidad, en caso de responder afirmativamente a la segunda cuestión que acabamos de presentar, de que una Comunidad Autónoma blindara su sistema financiero en previsión de futuras intervenciones de la Ley armonizadora o de modificaciones en la misma: en mi opinión, la respuesta acertadamente dada a esa cuestión, por el Prof. Pérez Royo, es negativa, supuesto el carácter irrenunciable de la competencia constitucional que se contempla en el art. 157.3, aspecto de la cuestión que, en mi opinión, refuerza las dudas que a la anterior he suscitado, desde la perspectiva factual de la existencia de esa Ley armonizadora y las funciones que le ha atribuido la JTC y, además y definitivamente, que no parece previsiblemente factible, en un espacio relativamente corto de tiempo, un escenario diverso del actual.

Por todo lo expuesto, no cabe sino suscribir en su totalidad la conclusión que alcanza el autor que nos conduce en estas reflexiones: ... los Estatutos de Au- 
tonomía deben limitarse, en relación con la Hacienda a los aspectos generales: la fijación de los principios sobre la financiación, en línea con lo que establece la Constitución, y a la regulación del diseño institucional de la Hacienda autonómica. Para concluir, con rotundidad, en lo que tal pareciere fuera una respuesta específica a la cuestión nuclear de estas Jornadas: Asumida la realidad de la LOFCA de un sistema común de financiación, las cuestiones relativas a su modificación deben ser separadas de las de la reforma estatutaria ${ }^{21}$.

\section{EL SISTEMA Y EL MODELO DE FINANCIACIÓN EN LOS NUE- VOS ESTATUTOS.}

Con la conclusión que acabamos de alcanzar podríamos dar por concluida esta intervención, mas aún considero de interés plantear, precisamente, cuáles han sido las líneas maestras que han fijado los nuevos Estatutos cuya reforma está más avanzada, es decir, cuáles han sido los principios que recogen sobre la financiación y cuál es el diseño institucional que realizan a fin de verificar si, efectivamente, se hace necesario una modificación del modelo vigente, incluso si es necesario, además, introducir modificaciones en la LOFCA. No esperen sin embargo, sino unas breves consideraciones sobre esta cuestión que, no obstante, considero crucial, sobre todo, para poner jurídicamente, al menos, en su sitio tantas voces y tantos ecos, como han acompañado el fatigoso alumbramiento de algunas de las reformas de esos textos estatutarios.

Mas, al tiempo, no será estéril referirse a las vías por donde, en cualquier caso, debiera discurrir la reforma del modelo. En el estado actual de la cuestión (o del arte), es ya un terreno definitivamente abonado insistir en la necesidad de incrementar la corresponsabilidad de las CCAA, lo que no viene a significar sino la necesidad de caminar por la senda de la intensificación de la autonomía financiera en el aspecto del ingreso, no sólo porque se arbitren medidas mediante las cuales éstas alleguen mayores recursos, sino porque éstos serán el resultado de una más intensa participación de las CCAA en la autoconfiguración de éstos.

Los análisis macroeconómicos, nos muestran que aún los recursos económicos generados por los tributos cedidos apenas alcanzan el $70 \%$ de los recursos de las CCAA, financiándose el resto con la apelación al denominado Fondo de Suficiencia; desde la perspectiva del incremento del grado de autonomía, se trataría de conseguir un incremento del primero de los recursos, que

${ }^{21}$ PÉREZ ROYO (2006), negrillas nuestras. 
fuera susceptible de cubrir la totalidad de las necesidades financiera de las CCAA. Parece evidente que la opción tiene dos salidas naturales: bien el incremento del porcentaje de los cedidos -en aquellos supuestos en los que no se alcance el $100 \%$ de la cesión-, bien en añadir nuevas figuras a la cesta de los ya cedidos, pareciendo más razonable la primera que la segunda -porque en este caso, se trataría del IS, con las dificultades técnicas que implicaría-. Por tanto, estamos hablando claramente de tres figuras fundamentales: IRPF, IVA e IIEE. Respecto de estos dos ya sabemos algo acerca de los problemas que presenta la idea de la cesión de alguna competencia normativa sobre ellos, por lo que estaríamos hablando, práctica y efectivamente, del IRPF.

Esta solución supone una cesión del IRPF cercana al 90\%, aunque con severas limitaciones, acerca de lo que pudiéramos denominar la estructura básica o esqueleto configurador del tributo, competencia que debería, en todo caso, quedar en manos del poder normativo del Estado.

El objetivo sería la absorción del importe actual del $F S$, lo que nos llevaría a una conclusión cercana al pleno objetivo de la corresponsabilidad fiscal. El FS tendría una función más residual, es decir, complementaría los recursos de aquellas CCAA que con esta solución aún no cubrieran el 100\% de sus necesidades financieras.

Esta propuesta coincide, en sus líneas generales, con las que se han hecho en diversos Informes y Estudios -sobre todo al hilo de la elaboración de la Memoria económica del Estatuto catalán-; el trabajo del profesor PÉREZ ROYO, que ha tenido la generosidad de dejarme consultar, aún si publicar, insiste en esta idea y apunta en la dirección de destinar los saldos negativos de las CCAA que logran un superávit con esta solución a financiar a aquéllas en las que ni siquiera con ésta lograran una financiación del 100\% de sus gastos.

Entre las primeras, señala el profesor PÉREZ ROYO a Baleares, Cataluña, Madrid y, posiblemente, Valencia. Si sus cálculos son fidedignos, se trataría de un importe en torno a los 11.000 millones de euros que se repartirían entre las Comunidades que no alcanzaran ese $100 \%$ de financiación de sus gastos.

Desde luego, el grado de autonomía financiera sería asimétrico, aunque con un abanico, siempre utilizando los datos ya citados, de entre el $75 \%$, para las segundas, al $100 \%$ para las primeras.

La solución haría más transparente el principio de solidaridad fiscal y exigiría un exquisito respeto al principio de lealtad institucional vertical y hori- 
zontal; sin olvidar, evidentemente, un difuso compromiso de mantener unos límites mínimos de exigencia de presión fiscal, que no produjera una viciada dejación del ejercicio de las competencias normativas, en relación con los impuestos cedidos, que pudiera concretarse en la exigencia de un mínimo relativo de esfuerzo fiscal, en la línea de lo que se exige en el ámbito local.

Un análisis conjunto de los tres Estatutos cuyo proceso de reforma está más avanzado da como resultado las siguientes conclusiones:

$\checkmark$ en primer lugar, los tres Estatutos reconocen la necesidad de la existencia de una ley armonizadora, por lo que el desarrollo de sus competencias financieras se hará siempre de acuerdo con la CE, el Estatuto y la LO a que hace referencia el art. 157.3 CE (arts. 67.3 EV; 201.1 EC; 177.1 EA).

$\sqrt{ } \quad$ los tres Estatutos parten del actual sistema de diferenciación entre tributos cedidos con competencias normativas plenas o parciales y sólo el Estatuto de Cataluña recoge en las DDAA $8^{\mathrm{a}}, 9^{\mathrm{a}}$ y $10^{\mathrm{a}}$ una modificación del statu quo actual que se concretaría en una nueva Ley de Cesión que, lógicamente, sería de aplicación a todas las CCAA (art. 73.2 EV y $175.3 \mathrm{EA}$ ), en la que las más importantes novedades estriban en

- un incremento del porcentaje de cesión del IRPF: será del 50\%, previéndose, asimismo, el aumento de las competencias normativas de la Comunidad en dicho Impuesto

- asimismo en la mencionada nueva Ley de Cesión, habrá de reconocerse un porcentaje de cesión del $58 \%$ en el rendimiento de los IIEE.

- por último, un porcentaje de cesión del $50 \%$ del rendimiento del IVA.

$\sqrt{ }$ en los tres Estatutos se hace referencia a la posibilidad de crear entes (Servicio Tributario Valenciano; Agencia Tributaria de Andalucía; Agencia Tributaria de Cataluña) que se hagan cargo directamente de la gestión de los tributos propios o cedidos totalmente. Asimismo se hace referencia a la constitución, en el plazo de dos años, de un Consorcio que pudiera constituir el germen de una denominada Administración Tributaria de Cataluña (art. 204 EC); a similares organismos se refieren los arts. 178.2 EA y, aunque de modo menos preciso, el art. 75.3 EV. 
$\checkmark$ el segundo pilar del sistema, qué duda cabe, no es sino el modo de verificarse el principio de solidaridad. Si nos atenemos a los cálculos que nos propone el Profesor PÉREZ ROYO, la totalidad de las CCAA verían reducida, lógicamente, las aportaciones recibidas mediante el $F S$ y el FCI, aunque la mayoría continuarían teniendo un saldo positivo, y sólo las de Baleares, Cataluña y Madrid y, tal vez, Valencia, lo tendrían negativo. Los Estatutos han hecho referencia a esta cuestión, siendo la más precisa la que se encuentra recogida en el EC, en su art. 206.3, en el que se hacen dos afirmaciones de suma importancia

- en primer lugar, se hará eco de lo establecido en el art. 158.3 CE, al referirse a un nivel similar en el conjunto del Estado en la prestación de los servicios esenciales, es decir, educación, sanidad y otros servicios esenciales del Estado del bienestar. Entre las variables a tener en cuenta, en la línea de carácter general que ya apuntamos más arriba, se hará referencia a la necesidad de que todas las CCAA del Estado lleven a cabo un esfuerzo fiscal similar.

- la segunda afirmación es bien parca, mas sumamente relevante: los mecanismos de nivelación y solidaridad serán fijados por el Estado.

- la determinación de los requisitos de ponderación de la equidad no son muy diversos de los que actualmente se establecen a fin de repartir el FS; se hablará en el art. 206.6 de población, rectificada por los costes diferenciales y por variables demográficas, por el porcentaje de población inmigrante, densidad de población, dimensión de los núcleos urbanos, etc., siendo, en este aspecto menos preciso, el EA en su art. 180.6 y 180.7.

No quiero extenderme más, aunque no sé si he respondido a las expectativas de los organizadores de estas Jornadas. Sigo pensando que la construcción del Estado complejo que se deriva del diseño constitucional no es la búsqueda de un objetivo preciso y concreto, de ahí la necesaria inconcreción de muchas cuestiones que están necesariamente teñidas de consideraciones de tiempo y lugar, es decir, que no son el resultado de la aplicación pura de normas jurídicas que, como hemos visto, a lo largo de nuestra andadura democrática de casi treinta años, han dado lugar a soluciones en este campo que, si se miran con suficiente perspectiva, son sencillamente diferentes, muy diferentes, sin que ello afecte necesariamente a ninguna pretendida esencia del Estado. 
Los modelos de financiación no sólo no son ajenos a estas consideraciones, sino que son un fiel reflejo de las mismas, es más, son el paradigma de ésas. Ésta fue una de las muchas ideas que nos enseñó el magisterio del profesor García-Añoveros y tal vez, además, no dejen de ser exquisitamente metodológicas.

Muchas gracias por su atención, les pido excusas por esta larga exposición de asuntos que, a buen seguro, tendrán respuestas más precisas de las que mi ingenio ha sido capaz, a lo largo del debate; me conformo con haber suscitado su curiosidad. Gracias, de nuevo.

\section{BIBLIOGRAFÍA}

Se reproducen sólo las referencias bibliográficas completas citadas a lo largo del trabajo.

ESCRIBANO, Francisco.

"Algunas propuestas metodológicas para la (re)construcción de un Derecho Financiero del siglo XXI", en I Jornada Metodológica Jaime García Añoveros sobre la metodología académica y la enseñanza del Derecho Financiero y Tributario, IEF. Madrid. 2002.

GARCÍA-AÑOVEROS, Jaime.

"El discurso del Método en el ámbito Hacendístico", en I Jornada Metodológica Jaime García Añoveros sobre la metodología académica y la enseñanza del Derecho Financiero y Tributario, IEF. Madrid. 2002.

- "Génesis y desarrollo del modelo de financiación de las Comunidades Autónomas” en AA.VV., Estado regional-Estado federal: La Financiación de las Comunidades Autónomas, Salamanca. Diputación. 1986.

MARTÍN QUERALT, JUan; LOZANO SERRANO, Carmelo; TEJERIZO LÓPEZ, José M.; CASADO OLLERO, Gabriel.

Curso de Derecho Financiero y Tributario. Madrid. Tecnos. 2005.

MONASTERIO ESCUDERO, Carlos

"El sistema de financiación autonómica 2002" en SALINAS JIMÉNEZ, Javier (dir.) El nuevo modelo de financiación autonómica (2002). Madrid. IEF. 2002. 
MONASTERIO ESCUDERO, Carlos; PÉREZ GARCÍA, Francisco; SEVILLA SEGURA, José V.; SOLÉ VILANOVA, Joaquim.

Informe sobre el actual sistema de financiación autonómica y sus problemas (Primera parte) PALAU 1424 (1994); (Segunda Parte) PALAU 1425 (1995).

MORA LORENTE, M.D.

Impuestos cedidos: implicaciones internas y comunitarias, Tirant lo Blanch, Valencia, 2004.

PÉREZ ROYO, Fernando.

"Las reformas estatutarias y el modelo de financiación" (en prensa).

QUINTANA FERRER, Esteban

Reforma de la Hacienda autonómica y Libro Blanco sobre financiación de las Comunidades Autónomas. Valencia, Tirant lo Blanch, 1996.

RAMALLO MASSANET, Juan y ZORNOZA PÉREZ, Juan

"Sistema y modelos de financiación autonómica" en Perspectivas del Sistema Financiero 51 (1995).

RODRÍGUEZ BEREIJO, Álvaro

"Un reflexión sobre el sistema general de la financiación de las Comunidades Autónomas" Revista Española de Derecho Constitucional 15 (1985).

RUIZ ALMENDRAL, Violeta

Impuestos cedidos y corresponsabilidad fiscal. Valencia. Tirant lo Blanch. 2004. 